\title{
THE UNIQUE REPRESENTATION OF A SELFADJOINT BOUNDED LINEAR FUNCTIONAL
}

\author{
CHING - YUN SUEN
}

\begin{abstract}
It is well known that every selfadjoint bounded linear functional on a $C^{*}$-algebra has a unique minimal decomposition [6, Theorem 3.2.5]. In this paper we prove that under some conditions a selfadjoint completely bounded linear map with a unique minimal decomposition is equivalent to the map with a unique commutant representation (up to unitary equivalence). Using the results, we generalize the Gel'fand-Naimark-Segal construction.
\end{abstract}

1. Introduction. Throughout this paper let $M_{n}$ denote the $C^{*}$-algebra of complex $n \times n$ matrices generated as a linear space by the matrix units $E_{i j}(i, j=1,2, \ldots, n)$ and $\mathscr{L}(H)$ the algebra of all bounded linear operators on a Hilbert space $H$. Let $A$ and $B$ be $C^{*}$-algebras and let $L: A \rightarrow B$ be a bounded linear map. If for the maps

$$
L \otimes I_{n}: A \otimes M_{n} \rightarrow B \otimes M_{n},
$$

one has that $\sup _{n}\left\|L \otimes I_{n}\right\|$ is finite, then $L$ is called completely bounded and we let $\|L\|_{\mathrm{cb}}$ denote the sup. The map $L$ is called positive provided that $L(a)$ is positive whenever $a$ is positive, and is called completely positive if $L \otimes I_{n}$ is positive for all $n$. If $L\left(a^{*}\right)=L(a)^{*}$, we call $L$ selfadjoint. Given $S \subseteq \mathscr{L}(H)$, we let $S^{\prime}$ denote its commutant. A selfadjoint completely bounded map $\psi$ has a minimal decomposition [11, p. 104] provided that $\psi$ can be expressed as a difference of completely positive maps $\phi_{1}$ and $\phi_{2}$ with $\|\psi\|_{\mathrm{cb}}=\left\|\phi_{1}\right\|+\left\|\phi_{2}\right\|$. From [13, Proposition 5.1], we know that there are some conditions to ensure the existence of a minimal decomposition but the decomposition is not unique [11, p. 107] in general. We also know that the commutant representation for a completely bounded map is not unique [5]. However, there are some cases in which we can find that a selfadjoint completely bounded map has a unique commutant representation (up to unitary equivalence) if and only if the map has a unique minimal decomposition. Applying the above results, we have that each selfadjoint bounded linear functional on a $C^{*}$-algebra has a unique commutant representation which generalizes the Gel'fand-Naimark-Segal construction. Finally, the author gratefully acknowledges several valuable conversations with Professor V. Paulsen.

2. The uniqueness problem. Let $\mathrm{CB}(A, \mathscr{L}(H))$ denote the vector space of completely bounded linear maps from $C^{*}$-algebra $A$ into $\mathscr{L}(H)$. Let $L \in \mathrm{CB}(A, \mathscr{L}(H)$ ),

Received by the editors October 10, 1984.

1980 Mathematics Subject Classification. Primary 46L05.

C1985 American Mathematical Society $0002-9939 / 85 \$ 1.00+\$ .25$ per page 
from $[5$, p. 7] we know that

$$
\|L\|=\inf \left\{\|\phi\|:\left[\begin{array}{ll}
\phi & L \\
L^{*} & \phi
\end{array}\right] \text { is completely positive }\right\}
$$

We will apply the following theorem [5, Theorem 2.10] later:

THEOREM 2.1. Let $L \in \mathrm{CB}(A, \mathscr{L}(H))$, then there exists a Hilbert space $K, a$ *-homomorphism $\pi: A \rightarrow \mathscr{L}(K)$, an isometry $V: H \rightarrow K$ and an operator $T \in \pi(A)^{\prime}$ such that $[\pi(A) V H]$ is dense in $K,\|T\|=\|L\|$ and $L=V^{*} T \pi V$. If $L=L^{*}$, then $\|L\|=\|L\|_{\mathrm{cb}}$ and $T=T^{*}$.

We shall refer to a representation of the form given by Theorem 2.1 as a commutant representation with isometry of $L[5, \mathrm{p}$. 13]. We shall denote it by $(\pi, V, T, K)$. Suppose that $L \in \mathrm{CB}(A, \mathscr{L}(H))$ and that $\left(\pi_{i}, V_{i}, T_{i}, K_{i}\right), i=1,2$, are two commutant representations of $L$. We call these representations unitarily equivalent provided that there exists a unitary $U: K_{1} \rightarrow K_{2}$ such that

$$
U V_{1}=V_{2}, \quad U \pi_{1}=\pi_{2} U \text { and } U T_{1}=T_{2} U
$$

From [5, Proposition 3.1], we have the following

Proposition 2.2. Let $L \in \mathrm{CB}(A, \mathscr{L}(H))$. Then there is a unique completely positive map $\phi: A \rightarrow \mathscr{L}(H)$ with $\|\phi\|=\|L\|$ such that the map

$$
\left[\begin{array}{ll}
\phi & L \\
L^{*} & \phi
\end{array}\right]: A \otimes M_{2} \rightarrow \mathscr{L}(H) \otimes M_{2}
$$

is completely positive if and only if $L$ has a unique representation $(\pi, V, T, K)$ ( up to unitary equivalence).

Theorem 2.3. Let $L=L^{*} \in \mathrm{CB}(A, \mathscr{L}(H))$ and $L\left(I_{A}\right)=k I$, then the following statements are equivalent:

(i) L has a unique minimal decomposition.

(ii) There is a unique completely positive map $\phi: A \rightarrow \mathscr{L}(H)$ with $\|\phi\|=\|L\|_{\mathrm{cb}}$ such that the map

$$
\left[\begin{array}{ll}
\phi & L \\
L & \phi
\end{array}\right]: A \otimes M_{2} \rightarrow \mathscr{L}(H) \otimes M_{2}
$$

is completely positive.

(iii) $L$ has a unique representation $(\pi, V, T, K)$ (up to unitary equivalence) with $T=T^{*}$ and $\|T\|=\|L\|_{\mathrm{cb}}$.

Proof. From Theorem 2.1 and Proposition 2.2, we know that (ii) and (iii) are equivalent. Suppose that (i) is true. By [5, Proposition 2.8], there is a completely positive map $\phi: A \rightarrow \mathscr{L}(H)$ with $\|\phi\|=\|L\|_{\text {cb }}$ such that the map

$$
\left[\begin{array}{ll}
\phi & L \\
L & \phi
\end{array}\right]: A \otimes M_{2} \rightarrow \mathscr{L}(H) \otimes M_{2}
$$


is completely positive. It is easy to see that $\frac{1}{2}(\phi+L)-\frac{1}{2}(\phi-L)$ is a minimal decomposition of $L$. Similarly, if there is another completely positive map $\tilde{\phi}$ : $A \rightarrow \mathscr{L}(H)$ with $\|\tilde{\phi}\|=\|L\|_{\mathrm{cb}}$ such that the map

$$
\left[\begin{array}{ll}
\tilde{\phi} & L \\
L & \tilde{\phi}
\end{array}\right]: A \otimes M_{2} \rightarrow \mathscr{L}(H) \otimes M_{2}
$$

is completely positive, then $\frac{1}{2}(\tilde{\phi}+L)-\frac{1}{2}(\tilde{\phi}-L)$ is also a minimal decomposition of $L$. By (i), we have that $\phi=\tilde{\phi}$. Hence (i) implies (ii). Conversely, if (ii) is true. By [13, Proposition 5.1], $L$ has a minimal decomposition $\psi_{1}-\psi_{2}$. Let $\psi=\psi_{1}+\psi_{2}$; by [10, Theorem 3.9], we know that

$$
\|L\|_{\mathrm{cb}}=\min \left\{\|\phi\|_{\mathrm{cb}}: \phi \pm L \text { are completely positive }\right\} .
$$

Since $\psi_{1}+\psi_{2} \pm L$ are completely positive, we have that

$$
\|L\|_{\mathrm{cb}}=\left\|\psi_{1}\right\|+\left\|\psi_{2}\right\| \leqslant\left\|\psi_{1}+\psi_{2}\right\| .
$$

Hence $\|\psi\|=\|L\|_{\mathrm{cb}}$. Let $\psi=\psi_{1}+\psi_{2}$; applying Stinespring's Theorem [9], $\psi$ has a minimal representation $V^{*} \pi(\cdot) V$. By [1, Theorem 1.4.2], $\psi_{i}$ has representation $V^{*} T_{i} \pi(\cdot) V$ with $0 \leqslant T_{i} \leqslant I$ and $T_{i} \in \pi(A)^{\prime}, i=1,2$. Hence

$$
L(\cdot)=V^{*}\left(T_{1}-T_{2}\right) \pi(\cdot) V \text { and } \quad-I \leqslant T_{1}-T_{2} \leqslant I .
$$

Since the matrix

$$
\left[\begin{array}{cc}
I & T_{1}-T_{2} \\
T_{1}-T_{2} & I
\end{array}\right]
$$

is positive, by [5, Proposition 2.6], the map

$$
\left[\begin{array}{ll}
\psi & L \\
L & \psi
\end{array}\right]: A \otimes M_{2} \rightarrow \mathscr{L}(H) \otimes M_{2}
$$

is completely positive. Similarly, if $L$ has another minimal decomposition $\tilde{\psi}_{1}-\tilde{\psi}_{2}$, then the map

$$
\left[\begin{array}{cc}
\tilde{\psi}_{1}+\tilde{\psi}_{2} & L \\
L & \tilde{\psi}_{1}+\tilde{\psi}_{2}
\end{array}\right]: A \otimes M_{2} \rightarrow \mathscr{L}(H) \otimes M_{2}
$$

is completely positive. By (ii), we have that $\tilde{\psi}_{1}+\tilde{\psi}_{2}=\psi_{1}+\psi_{2}$ and hence

$$
\tilde{\psi}_{1}=\frac{1}{2}\left(\tilde{\psi}_{1}+\tilde{\psi}_{2}+L\right)=\frac{1}{2}\left(\psi_{1}+\psi_{2}+L\right)=\psi_{1} .
$$

Therefore, (ii) implies (i).

REMARK 2.4. Let $L=L^{*} \in \mathrm{CB}(A, \mathscr{L}(H))$, from the same argument as Theorem 2.3 , we know that the map $\left[\begin{array}{ll}\phi & L \\ L\end{array}\right]$ is completely positive if and only if $\phi \pm L$ are completely positive.

EXAMPLE 2.5. Let $\theta(n)$ denote the transpose map of $M_{n}$ [13], then $\theta(n)$ has a unique minimal decomposition [11, Theorem 2.2] and $\|\theta(n)\|_{\mathrm{cb}}=n$ [13]. Now, let $\phi$ : $M_{n} \rightarrow M_{n}$ be the completely positive map defined by

$$
\phi\left(\left(a_{i j}\right)\right)=\operatorname{Tr}\left(\left(a_{i j}\right)\right) I_{n},
$$

then $\|\phi\|=\|\theta(n)\|_{\mathrm{cb}}=n$. It is not difficult to see that the matrix

$$
\left[\begin{array}{cc}
\phi\left(E_{i j}\right) & \theta(n)\left(E_{i j}\right) \\
\theta(n)\left(E_{i j}\right) & \phi\left(E_{i j}\right)
\end{array}\right]_{i j}
$$


is positive. Hence the map

$$
\left[\begin{array}{cc}
\phi & \theta(n) \\
\theta(n) & \phi
\end{array}\right]: M_{n} \otimes M_{2} \rightarrow M_{n} \otimes M_{2}
$$

defined by

$$
\left[\begin{array}{cc}
\phi & \theta(n) \\
\theta(n) & \phi
\end{array}\right]\left(\left[\begin{array}{ll}
a & b \\
c & d
\end{array}\right]\right)=\left[\begin{array}{cc}
\phi(a) & \theta(n)(b) \\
\theta(n)(c) & \phi(d)
\end{array}\right]
$$

is completely positive [3, Theorem 2]. By Theorem 2.3, we know that $\phi$ is unique. Hence $\frac{1}{2}(\phi+\theta(n))-\frac{1}{2}(\phi-\theta(n))$ is the unique minimal decomposition of $\theta(n)$. Furthermore, let $\Phi$ be the Choi's map defined by

$$
\Phi\left(\left(a_{i j}\right)\right)=\left\{(n-1) \operatorname{Tr}\left(\left(a_{i j}\right)\right)\right\} I_{n}-\left(a_{i j}\right)
$$

[11, Theorem 1.3], the structure of the segment between $\Phi$ and $\theta(n)$ is determined. By Theorem 2.3, each map in the segment has a unique commutant representation.

Corollary 2.6. Let $L=L^{*}: A \rightarrow C$ be a bounded linear map, then $L$ has a unique representation $V^{*} T \pi V$ (up to unitary equivalence) with $T \in \pi(A)^{\prime}, T=T^{*}$ and $\|T\|=\|L\|$.

Proof. From [8, Theorem 2.10], we know that $\|L\|=\|L\|_{\mathrm{cb}}$. By [6, Theorem 3.2.5], $L$ has a unique minimal decomposition. By Theorem 2.3, we have proved the corollary.

REMARK 2.7. In Corollary 2.6, for each $a \in A$, we have that $L(a)=$ $\langle\pi(a) T V(I), V(I)\rangle$. It is easy to see that $\pi$ is a cyclic representation and $V(I)$ is a unit cyclic vector. Hence, we have generalized the Gel'fand-Naimark-Segal construction: To each positive linear functional $\phi$, there corresponds uniquely, within unitary equivalence, a representation $(\pi, K)$ of $A$ with a cyclic vector $\xi$ such that $\phi(a)=$ $\langle\pi(a) \xi, \xi\rangle$.

We give an example of Corollary 2.6 in the following

EXAMPLE 2.8. Let $X$ be a compact Hausdorff space and let $C(X)$ be the $C^{*}$-algebra of continuous functions on $X$ with sup norm. Let $L$ be a selfadjoint bounded linear functional on $C(X)$; by the Riesz Theorem [7, Theorem 6.19], there corresponds a unique complex regular Borel measure $\mu$ such that $L(f)=\int_{X} f d \mu$. By [7, Theorem 6.12], there is a Borel function $h$ with $|h|=1$, such that

$$
d \mu=h d|\mu| \text { and }\|L\|=\|L\|_{\mathrm{cb}}=|\mu|(X) .
$$

Since $L$ is selfadjoint, we have that $h$ is real-valued $|\mu|$-a.e. Let $S=\{x \in X$ : $h(x)=1\}$, then

$$
L(f)=\int_{S} f d|\mu|-\int_{X-S} f d|\mu| .
$$

Let $\phi: C(X) \rightarrow C$ be the positive linear map defined by

$$
\phi(f)=\int_{X} f d|\mu| .
$$


It is easy to see that $L$ has a minimal decomposition $\frac{1}{2}(\phi+L)-\frac{1}{2}(\phi-L)$. By Theorem 2.3 and Remark 2.4, we know that $\phi$ is the unique positive linear map with $\|\phi\|=|\mu|(X)$ such that the map

$$
\left[\begin{array}{ll}
\phi & L \\
L & \phi
\end{array}\right]: C(X) \otimes M_{2} \rightarrow M_{2}
$$

is completely positive. Let $V: C \rightarrow L_{2}(X ;|\mu|)$ be the operator defined by $\lambda \rightarrow \lambda I$ and $\pi: C(X) \rightarrow \mathscr{L}\left(L_{2}(X ;|\mu|)\right)$ be the ${ }^{*}$-representation defined by $\pi(f)=M_{f}$. It is not difficult to see that

$$
\phi(f)=\tilde{V}^{*} \pi(f) \tilde{V}=\int_{X} f d|\mu|
$$

and $[\pi(C(X)) \tilde{V}(1)]$ is dense in $L_{2}(X ;|\mu|)$. Let $g: C(X) \rightarrow C$ be the map defined by

$$
g(x)= \begin{cases}1 & \text { if } x \in S, \\ -1 & \text { if } x \in X-S,\end{cases}
$$

then $M_{g} \in \pi(C(X))^{\prime}, M_{g}=M_{g}^{*},\left\|M_{g}\right\|=1$, and

$$
\tilde{V}^{*} M_{g} \pi(f) \tilde{V}=\int_{X} g f d|\mu|=\int_{S} f d|\mu|-\int_{X-S} f d|\mu|=L(f) .
$$

Hence, $L$ has a unique commutant representation $V^{*} T \pi V$, where $V=\tilde{V} / \sqrt{|\mu|(X)}$ and $T=|\mu|(X) M_{g}$.

\section{REFERENCES}

1. W. B. Arveson, Subalgebras of $C^{*}$-algebras, Acta. Math. 123 (1963), 141-224.

2. M. D. Choi, Positive linear maps on $C^{*}$-algebras, Canad. J. Math. 24 (1972), 520-529.

3. Completely positive linear maps on complex matrices, Lincar Algebra and Appl. 10 (1975), 285-290.

4. V. I. Paulsen, Every completely polynomially bounded operator is similar to a contraction, J. Funct. Anal. 55 (1984), 1-17.

5. V. I. Paulsen and C. Y. Suen, Commutant representations of completely bounded maps, J. Operator Theory 13 (1985), 87-101.

6. G. K. Pederson, $C^{*}$-algebras and their automorphism groups, Academic Press, London, 1979.

7. W. Rudin, Real and complex analysis, McGraw-Hill, New York, 1966.

8. R. R. Smith, Completely bounded maps between $C^{*}$-algebras, J. London Math. Soc. 27 (1983), 157-166.

9. W. F. Stinespring, Positive functions on $C^{*}$-algebras, Proc. Amer. Math. Soc. 6 (1955), 211-216.

10. C. Y. Suen, The representation theory of completely bounded maps on $C^{*}$-algebras, Thesis, University of Houston, 1983.

11. T. Takasaki and J. Tomiyama, On the geometry of positive maps in matrix algebras, Math Z. 184 (1983), 101-108.

12. J. Tomiyama, On the transpose map of matrix algebras, Proc. Amer. Math. Soc. 88 (1983), 635-638. . Recent development of the theory of completely bounded maps between $C^{*}$-algebras (preprint).

Department of Mathematics, Texas A \& M University at Galveston, Galveston. Texas 77553 